\title{
Paper
}

\section{Selective Area Growth of III-Nitride and Theilr Application for Emitting Devices}

\author{
Kazumasa HIRAMATSU, Hideto MIYAKE and Da-Bing LI
}

Department of Electrical and Electronic Engineering, Mie University

Received December 14, 2007, Accepted February 26, 2008

\begin{abstract}
In this paper, the high quality AIGaN with high-Al content was grown on in-situ monitoring controlled selective area growth (SAG) GaN and the dislocation density of $A I G a N$ is $1-3 \times 10^{8} \mathrm{~cm}^{-2}$. Furthermore, we use SAG to fabricate InGaN/GaN pyramid structures. CL (Cathodoluminescence) measurements reveal that the thickness, $\mathrm{CL}$ peak wavelength and $\mathrm{CL}$ intensity gradually increased from the bottom to the top of the facet. Furthermore, Vacuum fluorescent display (VFD) based on InGaN/GaN pyramid structures was demonstrated.
\end{abstract}

KEYWORDS: Selective area growth, AIGaN, InGaN/GaN pyramid structuresVacuum fluorescent display

\section{Introduction}

For III-nitride semiconductors, the selective growth (SAG) and the epitaxial lateral overgrowth (ELO) are promising techniques to grow high-quality epitaxial film and to fabricate various semiconductor microstructures such as pyramids, quantum wires, and quantum dots. This structural control technique greatly improve electronic and optoelectronic device perform, for example, prolonging laser diode (LD) lifetime ${ }^{1)}$, enhancing ultraviolet (UV) light emitting diode (LED) external quantum efficiency ${ }^{2}$, and decreasing HFETs gate leakage ${ }^{3)}$.

In recent years, SAG and ELO techniques have been well developed as follows: utilization of other masks as tungsten ${ }^{45)}$ followed by air-bridge ELO ${ }^{677}$, PENDEO epitaxy $^{8}$ followed by grooved strip structure ${ }^{9}$ and structure substrate ${ }^{210111)}$, hetero ELO'12, Facet Controlled ELO (FACELO) 13)14), self-organized islands ${ }^{15116)}$ followed by nanoscale lateral epitaxial overgrowth (NLEO)17), and in situ SiN nano"mask ${ }^{18}$. For GaN, these novel structural control techniques can effectively decrease dislocation density and improve crystal quality. However, for other III-nitride semiconductors such as AlGaN, these structural control techniques don't always affect. The main cause lies on that AlGaN has smaller lattice constant than $\mathrm{GaN}$, which induces tensile stress and easily make film cracking during common SAG and ELO. In addition, Al has highly chemical activity and AlGaN tend to form polycrystals on the mask. These disadvantages make it difficult to obtain high quality AlGaN with SAG and ELO.

In this paper, in-situ monitoring controlled SAG is developed to achieve AlGaN ELO and obtain high quality
AlGaN with dislocation density of $1-3 \times 10^{8} \mathrm{~cm}^{2}$. Meanwhile, SAG is applied to fabricate InGaN/GaN pyramid structures, which is potential to be used to fabricate VFD operated at low-voltage. And the facet dependence of the optical properties of InGaN is investigated by CL.

\section{Experimental}

A low-pressure metalorganic vapor phase epitaxy (LPMOVPE) is used to grow SAG- AlGaN and InGaN/GaN pyramid structures. (0001) sapphire is used as the substrate. Ammonia ( $\left.\mathrm{NH}_{3}\right)$, trimethylgallium ( $\mathrm{TMG}$ ), trimethylaluminum (TMAl), and trimethylindium (TMI) were used as source materials. Monomethylsilane $\left(\mathrm{CH}_{3} \mathrm{SiH}_{3}\right)$ was used as a dopant source to obtain high conductivity.

The growth procedures were as follows: For SAG AlGaN, a $1.0 \mu m$ thick (0001) AlN/sapphire was used as a substrate. After the deposition of a $100 \mathrm{~nm}$-thick $\mathrm{SiO}_{2}$ film by RF sputtering on the underlying Si-doped GaN, stripe patterns were fabricated by a conventional photolithographic method. The $\mathrm{SiO}_{2}$ mask had windows with a $7 \mu \mathrm{m}$ diameter and $3 \mu \mathrm{m}$ spacing. Then, a Si-doped GaN layer was grown at $1080^{\circ} \mathrm{C}$ at a pressure of 50 Torr. The grow time Growth time is determined by in-situ monitoring. Finally, AlGaN was grown at $1180^{\circ} \mathrm{C}$ at a pressure of 60 Torr.

For InGaN/GaN pyramid structures: $3-\mu \mathrm{m}$-thick $\mathrm{Si}-$ doped GaN film was grown on a sapphire (0001) substrate. Then Si-doped GaN was patterned with a mask for pyramid structures. Subsequently, Si-doped GaN with pyramid structures was grown on the patterned underlayer by MOVPE. Finally, Si-doped InGaN was 
grown at $780^{\circ} \mathrm{C}$ with various growth times from $3 \mathrm{~min}$ to $27 \mathrm{~min}$.

A high resolution X-ray diffraction (HRXRD) was used to study the crystal quality. And a scanning electron microscope (SEM) with a low-temperature CL setup was used to investigate the optical properties. The CL system provides SEM images, monochromatic CL images and CL wavelength images during one scan.

\section{Results and discussion}

3.1 AlGaN grown on in-situ monitoring controlled SAG $\mathrm{GaN}$

Figure 1 is the sketch of SAG AlGaN: GaN with $\{11-20\}$ facet was fabricated on the pattern $\mathrm{AlN}$, which was used to SAG AlGaN. Before GaN coalescent, unmerged GaN layer can reduce dislocation density and release tensile stress; however, after GaN coalescent, GaN had the larger lattice constant than AlGaN, which induced tensile stress and easily crack $\mathrm{AlGaN}$. Then, GaN coalescent process has to be controlled during SAG.

For this purpose, an in-situ monitoring based on reflecting light intensity is set up to control GaN coalescent process. During GaN growth, the morphology changes from isolated small islands via larger islands to a smooth surface (see Figure 2). Before GaN coalescent, incident light is diffuse reflected on the surface, which cause the reflecting light intensity weak. During GaN coalescent, these isolated small islands merge into larger islands so that reflecting light intensity increases rapidly. At the end of GaN coalescent, mirror reflection is dominated and reflecting light intensity becomes saturation. Then, this in situ monitor can reveal the GaN facet formation and help us to control coalescent process. In our experiments, after $65 \mathrm{~min} \mathrm{GaN}$ growth, reflecting

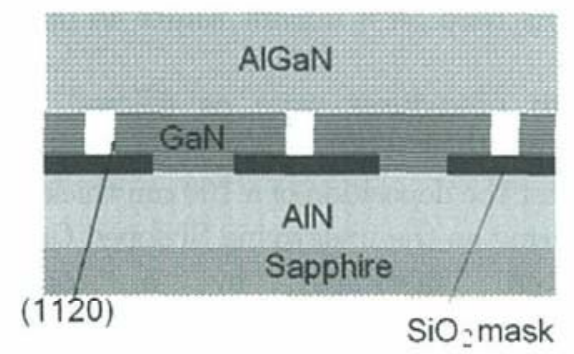

Figure 1 Schematic diagram of AIGaN ON SAG GaN MODEL

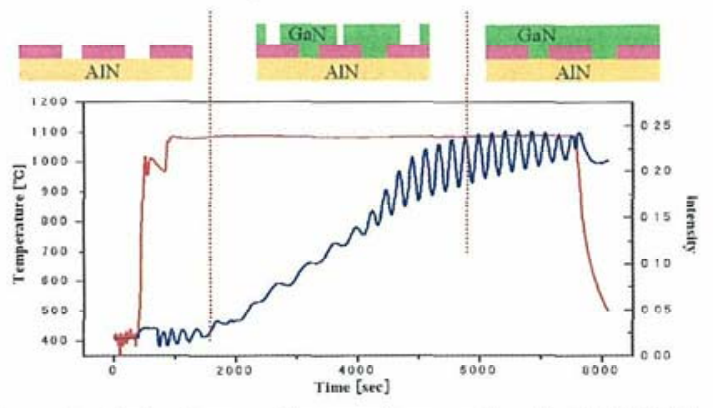

Figure 2 Reflection and temperature profiles for SAG GaN light intensity became saturation and the $\mathrm{GaN}$ was near to entire coalescent.

Figure 3 is scanning electron microscopy (SEM) images for SAG GaN. The lateral face of stripe was (11-20), kept about 1 2 $\mathrm{m}$ distance from each other and the height was $3 \mu \mathrm{m}$. Here, When $\varphi=0^{\circ}$ (the incidence direction of Xray is parallel to stripe direction ), full width at half maximum (FWHM) of (0002) GaN X-ray rocking curves (XRC) was 249 arc sec, and FWHM of (10-12) GaN XRC was 360 arc sec; When $\varphi=90^{\circ}$ (the incidence direction of $\mathrm{X}$-ray is perpendicular to the stripe direction ), the FWHM value of (0002) GaN XRC was 288 arc sec, and FWHM of $(10-12)$ XRC GaN was 490 arc sec.

After AlGaN growth, the SEM image is exhibited in Fig.4. The sample exhibited an island-like and smooth morphology. When $\varphi=0^{\circ}$, the FWHM of (0002) XRC AlGaN was 457 aresec, and FWHM of (10-12) XRC AlGaN was 1164 arc sec; when $\varphi=90^{\circ}$, FWHM of (0002) AlGaN XRC was 843 arcsec, and FWHM of (10-12) AlGaN XRC was 1177 arcsec. And the surface CL measurement revealed that dislocation density is $2.9 \times 10^{8}$ $\mathrm{cm}^{-2}$, which was two orders of magnitude lower than that of conventional $\mathrm{AlGaN}$ grown on $\mathrm{AlN} /$ sapphire template (Figure 5).

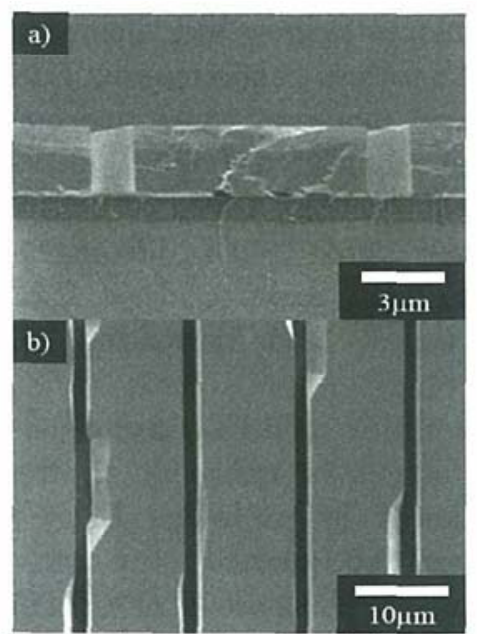

Figure 3 Cross-sectional and surface SEM images for SAG GaN

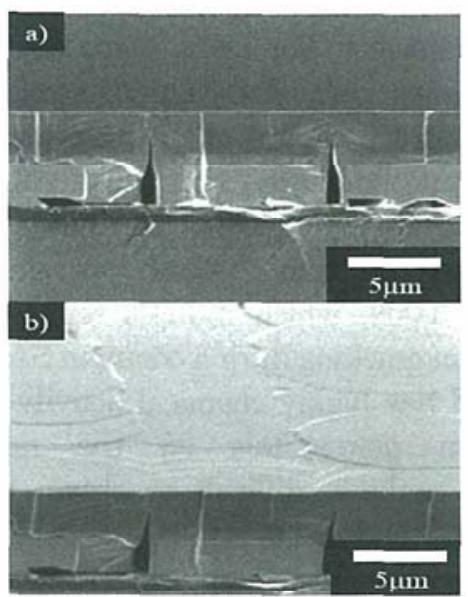

Figure 4 Cross-sectional and bird-eyes view SEM images for AlGaN grown on SAG GaN 

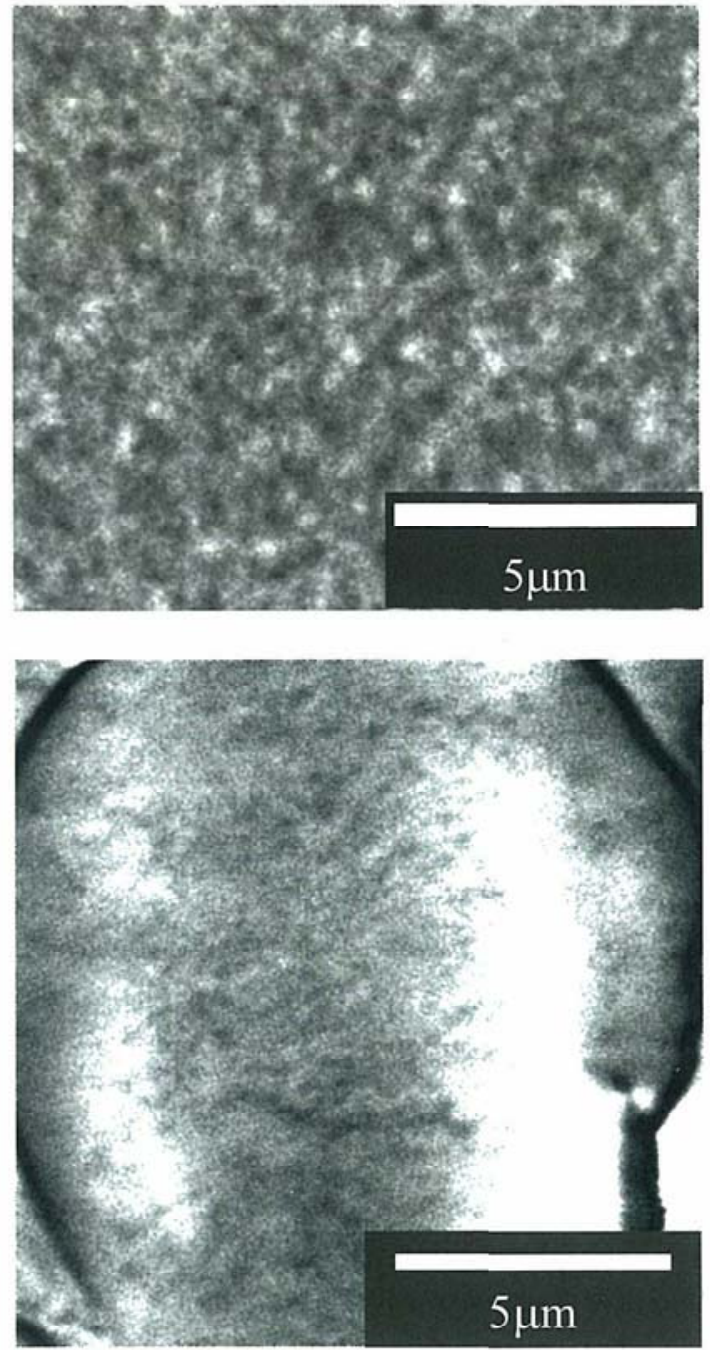

Figure $5 \mathrm{CL}$ mapping of AlGaN grown on flat AIN (up) and SAGGaN/AIN/sapphire (bottom)

\subsection{InGaN on GaN Facets}

Using the dot pattern mask, the facet structure of GaN depends on the growth temperature ${ }^{19220)}$, TMG flow rate ${ }^{19)}$, and $\mathrm{NH}_{3}$ partial pressure ${ }^{20}$. Under the growth condition of a high V/III source gas ratio, a surface that has N-polarity is stabilized, and it becomes more stable at higher pressures or lower temperatures, so that the growth rate tends to decrease, resulting in the $\{1-101\}$ facet. However, under the opposite growth conditions of lower pressures or higher temperatures, the $\{1-101\}$ facet becomes unstable because the surface nitrogen atoms are not stabilized, resulting in the $\{1-100\}$ and (0001) facets, which are energetically more favorable. Under our growth condition, the $\{1-101\}$ facet is more stable. Figure 6(a) shows an SEM image of InGaN on GaN hexagonal pyramid structures. The facet of pyramids had a slope of about $62^{\circ}$ with respect to the c-plane corresponding to the $\{10-11\}$ facet.The height of pyramids was about $8 \mu \mathrm{m}$. The thickness of InGaN gradually increased from the lower part to the upper part of the facet. The thickness of the sample with $7 \mathrm{~min}$ growth of InGaN was observed to be

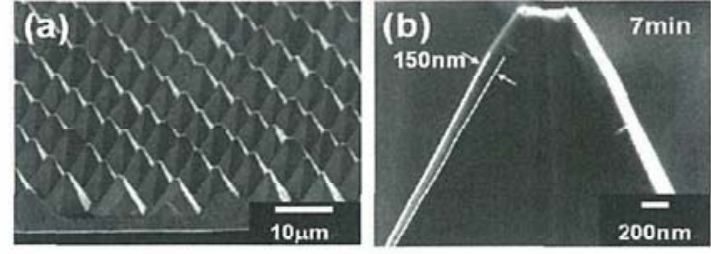

Figure 6 SEM images of InGaN on GaN facets (a) bird-eyes view image and (b) cross-section image(growth during for $7 \mathrm{~min}$ )

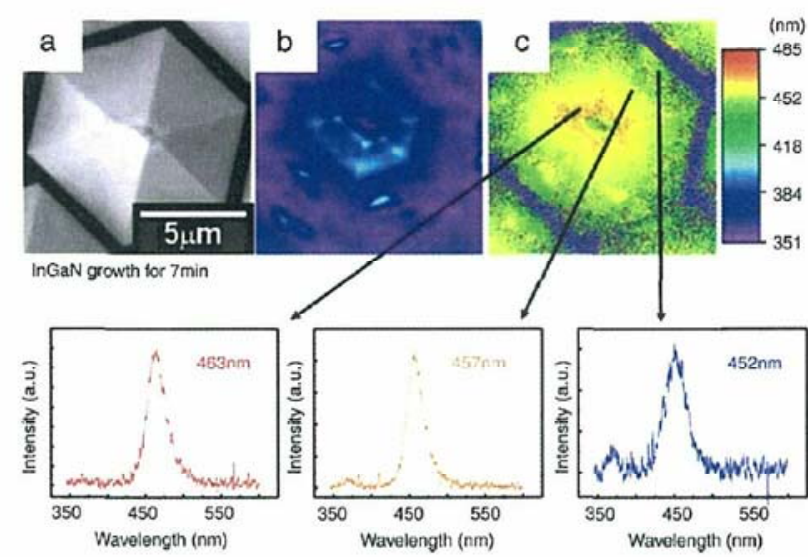

Figure 7 SEM images of InGaN on GaN facets

(a) bird-eyes view image and (b) cross-section image(growth during for $7 \mathrm{~min}$ )

about $150 \mathrm{~nm}$ at the top of the facet but was not measurable in the bottom region, as shown in Figure 6(b).

Figure $7 \mathrm{a}-\mathrm{c}$ show SEM images, a CL wavelength image and a CL intensity image of the surface for an InGaN/GaN pyramid structure of the sample with $7 \mathrm{~min}$ growth of InGaN. The GaN underlayer region showed a peak wavelength at about $365 \mathrm{~nm}$, while a peak wavelength at about $450 \mathrm{~nm}$ was observed in the region of the InGaN/GaN pyramid structure. CL spectra of the pyramid structure region shifted to a longer wavelength in the upper part of the facet. The upper part of the facet showed a peak wavelength at $463 \mathrm{~nm}$ and the bottom of the facet showed that of $452 \mathrm{~nm}$. In addition, CL intensity gradually increased from the lower part to the upper part of the facet.

Figure 8 shows the dependence of the CL intensity and peak wavelength on InGaN growth times. The CL intensity was enhanced with increasing InGaN growth time up to $14 \mathrm{~min}$. With a further increase of the InGaN growth time, the CL intensity decreased. It could be assumed that the thicker film resulted in a deterioration of the crystal quality.

Figure 9 displays the dependence of the CL peak wavelength on the position from the top to the bottom of the InGaN facet with different growth times. When the InGaN growth time was more than $27 \mathrm{~min}$, the distribution of the wavelength from the top to the bottom of the facet was almost constant. No marked, remarkable 


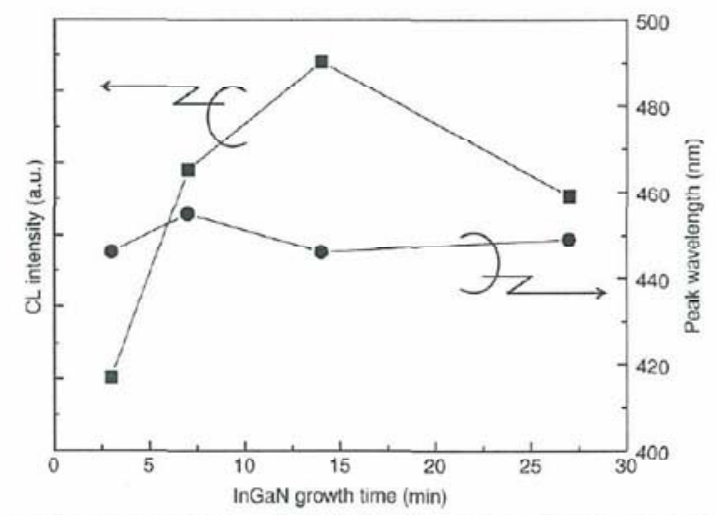

Figure 8 Dependence of $\mathrm{CL}$ intensity and peak wavelength on In $\mathrm{GaN}$ growth time

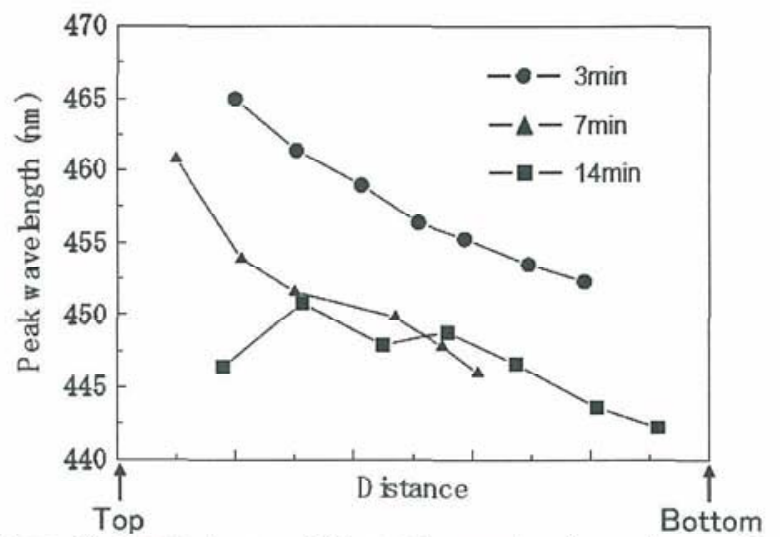

Figure 9 Dependence of $\mathrm{CL}$ peak wavelength on the position from the top to the bottom of the facet with different InGaN growth times

CL peak wavelength shift was observed for the sample with 27 min growth of InGaN.

Here, two possible mechanisms are proposed. One is that gallium and indium atoms diffuse towards the top of the facet. Nishizawa et al. have also reported an emission of InGaN/GaN multi-quantum-well (MQW) structures with the $\{11-22\}$ facet and found that gallium and indium atoms diffused to the top of the facet ${ }^{21}$. As we know, the $\mathrm{In}-\mathrm{N}$ bond is longer and weaker than the $\mathrm{Ga}-\mathrm{N}$ bond, and the indium atom preferentially diffuses to the top. Therefore, the upper part of the facet indicats a longer wavelength. For the sample with 27 min growth of $\mathrm{InGaN}$, the diffusion was further enhanced, and a facet with a slope larger than that of the $\{10-11\}$ facet appeared in the upper part of the facet with the greater thickness. Therefore, the indium diffusion towards the top of the facet was prevented and no CL peak shift was observed.

The other mechanism is the indium composition pulling effect. Shimizu et al. reported that the indium composition was reduced in a thin InGaN film grown on $\mathrm{GaN}^{22}$. It was suggested that indium atoms are excluded from the InGaN lattice during the early growth stages to reduce the deformation energy of the lattice mismatch. It was assumed that the growth rate at the upper part of the

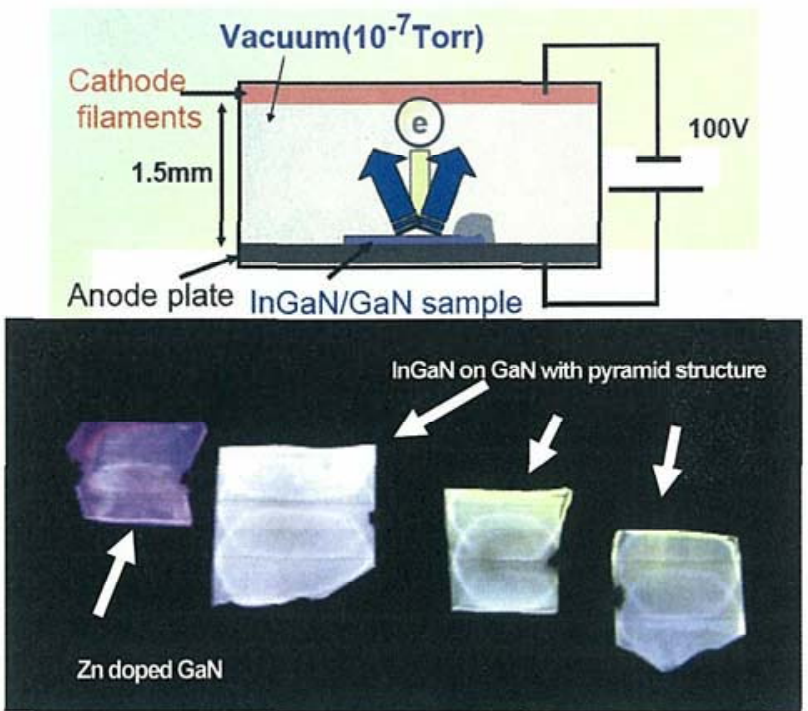

Figure 10 Sketch of VFD application

facet was faster than that at the bottom because of some source gas flow effects. In this case, the shorter wavelength at the bottom of the facet is due to the indium composition pulling effect. It was believed that the lack of an obvious peak shift for the sample with 27 min growth of InGaN was due to the greatly increased film thickness.

Meanwhile, we offer a prototype of Vacuum Fluorescent Display (VFD) with InGaN/GaN pyramid structures with acceleration voltage of $40-100 \mathrm{~V}$ and $\mathrm{Zn}$-doped $\mathrm{GaN}$, as shown in Fig.10. The results clearly demonstrate that bright VFD with InGaN/GaN pyramid structures can be operated at voltage as low as $100 \mathrm{~V}$.

\section{Concluding remark}

We develop an in-situ controlled SAG to grow other semiconductor besides $\mathrm{GaN}$. With this technique, we obtain high-quality $\mathrm{AlGaN}$ with dislocation density of 1$3 \times 10^{8} \mathrm{~cm}^{-2}$, which are two orders of magnitude lower than that of conventional AlGaN. The reduction of TD density is due to strain relaxation and ELO of AlGaN.

Furthermore, we use SAG to fabricate InGaN/GaN pyramid structures. For samples with InGaN growth time less than $27 \mathrm{~min}$, a redshift of the CL peak wavelength and enhancement of the CL peak intensity were observed from the lower part to the upper part of the facet. Perhaps, it can be attribute to indium diffusion mechanism or the indium composition pulling effect. For the sample with 27 min growth of InGaN, no obvious change in the CL peak wavelength or intensity was observed because of the hampered indium diffusion. This structure is used to fabricate VFD.

\section{Acknowledgements}

This work was partially supported by Grant-in-Aids for Scientific Research (No.18069006, 18060008, 18560010 
and 1806113) from Ministry of Education, Culture, Sports, Science and Technology and Japan Society for the Promotion of Science and Akasaki Research Center in Nagoya University.

\section{References}

(1) S. Nagahama, N. Iwasa, M. Senoh, T. Matsushita, Y. Sugimoto, H. Kiyoku, T. Kozai, M. Sano, H. Matsumura, H. Umemoto, K. Chocho and T. Mukai: High-Power and Long-Lifetime InGaN MultiQuantum-Well Laser Diodes Grown on Low Dislocation-Density GaN Substrates, Jpn. J. Appl. Phys, 39,pp.L647-L650 (2000).

(2) K. Tadatomo, H. Okagawa, Y. Ohuchi, T. Tsunekawa, Y. Imada, M. Kato and T. Taguchi:High Output Power InGaN Ultraviolet Light-Emitting Diodes Fabricated on Patterned Substrates Using Metalorganic Vapor Phase Epitaxy, Jpn. J. Appl. Phys, 40, pp.L583-L585 (2001).

(3) R. Ventury, H. Marchand, G. Parish, P. T. Fini, J. P. Tbbetson, S. Keller, J. S. Speck, S. P. DenBaars and U. K. Mishra, Compound Semiconductors (Inst. Phys. Conf. Ser. 162) ed H Sakaki et al. (1994) (Bristol: Institute of Physics Publishing) p. 177.

(4) Y. Kawaguchi, S. Nambu, H. Sone, T. Shibata, H. Matusushima, M. Yamaguchi, H. Miyake , K. Hiramatsu and N. Sawaki: Selective Area Growth of GaN Using Tungsten Mask by Metalorganic Vapor Phase Epitaxy, Jpn. J. Appl. Phys, 37, pp.L845-L848 (1998).

(5) H. Sone, S. Nambu, M. Yamaguchi, H. Miyake, K. Hiramatsu, Y. Iyechika, T. Maeda and N. Sawaki: Optical and Crystalline Properties of EpitaxialLateral-Overgrown-GaN Using Tungsten Mask by Hydride Vapor Phase Epitaxy,Jpn. J. Appl. Phys, 38, pp.L356-L359 (1999).

(6) I. Kidoguchi, A. Ishibashi, G. Sugahara and Y. Ban: Air-bridged lateral epitaxial overgrowth of GaN thin films, Appl. Phys. Lett., 76, pp.3768-3770 (2000).

(7) I. Kidoguchi, A. Ishibashi, G. Sugahara , A. Tsujimura and Y. Ban: Improvement of Crystalline Quality in GaN Films by Air-Bridged Lateral Epitaxial Growth, Jpn. J. Appl. Phys, 39, pp.L453* L456(2000).

(8) T. S. Zheleva, S. A. Smith, D. B. Thomson, T. Gehrke, K. J. Linthicum, P. Rajagopal, E. Carlson, W. M. Ashmawi and R. F. Davis: Pendeo-Epitaxy - A New Approach for Lateral Growth of GaN Structures, Mat. Res. Soc. Symp. Proc, 537, pp.G3.38- (1999).

(9) M. Ishida , H. Okagawa, K. Orita, O. Imafuji, M. Yuri, T. Sugino and K. Itoh: Drastic reduction of threading dislocation in GaN regrown on grooved stripe structure, J. Cryst. Growth, 221, pp.345-349(2000).
(10) T. Detchprohm, M. Yano, S. Sano, R. Nakamura, S. Mochiduki, T. Nakamura, H. Amano and I. Akasaki: Heteroepitaxial Lateral Overgrowth of $\mathrm{GaN}$ on Periodically Grooved Substrates: ANew Approach for Growing Low-Dislocation-Density GaN Single Crystals, Jpn. J. Appl. Phys, 40, pp.L16-L19 (2001).

(11) C. Ashby, C. Mitchel, J. Han, N. Missert, P. Provencio, D. Follstaedt, G. Peake, L. Griego: Low-dislocationdensity $\mathrm{GaN}$ from a single growth on a textured substrate, Appl. Phys. Lett. 77 pp.3233-3235 (2000).

(12) X. Zhang, R. R. Li, P. D. Dapkus, and D. H. Rich: Direct lateral epitaxy overgrowth of $\mathrm{GaN}$ on sapphire substrates based on a sparse $\mathrm{GaN}$ nucleation technique, Appl. Phys. Lett., 77,pp 2213-2215 (2000).

(13) K. Hiramatsu, K. Nishiyama, A. Motogaito, H. Miyake, Y. Iyechika, and T. Maeda: Recent Progress in Selective Area Growth and Epitaxial Lateral Overgrowth of III-Nitrides: Effects of Reactor Pressure in MOVPE Growth, Phys. Status Solidi a., 176, pp.535-543 (1999).

(14) B. Beaumont, V. Bousquet, P.Vennéguèes, M. Vaille, A. Bouillé, P. Gibart, S. Dassonneville, A. Amokrane, and B. Sieber: A Two-Step Method for Epitaxial Lateral Overgrowth of GaN, Phys. Status Solidi a,pp.567-571 176 (1999).

(15) S. Tanaka, M. Takeuchi, Y. Aoyagi: Anti-Surfactant in III-Nitride Epitaxy-Quantum Dot Formation and Dislocation Termination,Jpn. J. Appl. Phys, 39, pp.L831-L834 (2001).

(16) S. Sakai, T. Wang, Y. Morishima, and Y. Naoi: A new method of reducing dislocation density in GaN layer grown on sapphire substrate by MOVPE, J. Crystal Growth, 221, pp.334-337 (2000).

(17) K. Y. Zang, Y. D. Wang, and S. J. Chua, and L. S. Wang:Nanoscale lateral epitaxial overgrowth of GaN on Si (111), Appl. Phys. Lett. 87, pp.193106 (2005).

(18) H. Fitouri, Z. Benzarti, I. Halidou, T. Boufaden, and B. El Jani: Laser-reflectometry monitoring of the GaN growth by MOVPE using SiN treatment: study and simulation, phys. stat. sol. (a), 202, pp.2467-2473 (2005).

(19) S. Kitamura, K. Hiramatsu, N. Sawaki: Fabrication of GaN Hexagonal Pyramids on Dot-Patterned GaN/Sapphire Substrates via Selective Metalorganic Vapor Phase Epitaxy, Jpn. J. Appl. Phys., 34, pp.L1184-L1186 (1995).

(20) T. Akasaka, Y. Kobayashi, S. Ando, N. Kobayashi, M. Kumagai, J. Crystal Growth 72 (1998) 189.

(21) K. Nishizuka, M. Funato, Y. Kawakami, Y. Narukawa and T. Mukai:Efficient rainbow color luminescence from $\mathrm{In}_{\mathrm{x}} \mathrm{Gra1}_{\mathrm{x}} \mathrm{N}$ single quantum wells fabricated on $\{11 \overline{2} 2\}$ microfacets, App. Phys. Lett., 87,pp.231901(2005).

(22) M. Shimizu, Y. Kawaguchi, K. Hiramatsu and N. 
Sawaki: MOVPE growth of thick homogeneous InGaN directly on sapphire substrate using AlN buffer layer, Solid-State. Electron.,41,pp.145-147 (1997).

This paper is based on the authors' presentation given at the 1st International Conference on White LEDs and Solid State Lighting held in Tokyo, Japan, on November 26th - 30th, 2007. 